\title{
Influence of the geometric configuration of accretion flow on the black hole spin dependence of relativistic acoustic geometry
}

\author{
Pratik Tarafdar \\ S. N. Bose National Centre For Basic Sciences \\ Block-JD, Sector III, Salt Lake, Kolkata, India. \\ pratik.tarafdar@bose.res.in \\ Tapas Kumar Das \\ Harish-Chandra Research Institute \\ Chhatnag Road, Jhunsi, Allahabad, India. \\ tapas@hri.res.in
}

\begin{abstract}
Linear perturbation of general relativistic accretion of low angular momentum hydrodynamic fluid onto a Kerr black hole leads to the formation of curved acoustic geometry embedded within the background flow. Characteristic features of such sonic geometry depend on the black hole spin. Such dependence can be probed by studying the correlation of the acoustic surface gravity $\kappa$ with the Kerr parameter $a$. The $\kappa-a$ relationship further gets influenced by the geometric configuration of the accretion flow structure. In this work, such influence has been studied for multitransonic shocked accretion where linear perturbation of general relativistic flow profile leads to the formation of two analogue black hole type horizons formed at the sonic points and one analogue white hole type horizon which is formed at the shock location producing divergent acoustic surface gravity. Dependence of the $\kappa-a$ relationship on the geometric configuration has also been studied for monotransonic accretion, over the entire span of the Kerr parameter including retrograde flow. For accreting astrophysical black holes, the present work thus investigates how the salient features of the embedded relativistic sonic geometry may be determined not only by the background space-time, but also by the flow configuration of the embedding matter.
\end{abstract}

Keywords: Accretion disc, black hole physics, hydrodynamics, analogue gravity 


\section{Introduction}

Linear perturbation of inhomogenous inviscid transonic fluid leads to the formation of black hole like acoustic space time, characterized by an acoustic metric possessing some horizon-embedded features of the fluid flow Unruh [1981], Visser 1998|, Barceló et al. 2005], Unruh and Schützhold 2007]. Recent works Abraham et al. 2006], Das et al. [2007], Pu et al. [2012] demonstrate the emergence of such acoustic geometry and corresponding multiple acoustic horizons - one white hole type and two black hole type - within the general relativistic accretion of matter onto astrophysical black holes, either of the Schwarzschild or of the Kerr type. In these works, however, only a particular type of flow geometry (either flow with constant disc thickness or flow in hydrostatic equilibrium along the vertical direction) has been considered to study such emergent gravity phenomena. Since there might be three different geometric configurations with which matter may accrete onto astrophysical black holes (see, e.g. section 4 of Bilić et al. 2014], for the details of such configurations), it is necessary to observe how the characteristic features of the embedded acoustic metric get influenced by the flow geometry of the accreting matter. Tarafdar and Das 2015] have studied the polytropic as well as the isothermal relativistic accretion onto a non-rotating black hole for the three different geometric configurations, viz.flow with constant thickness, flow with quasi-spherical configuration and flow in hydrostatic equilibrium along the vertical direction ('constant height flow' $(\mathrm{CH})$, 'conical flow' (CF) and 'vertical equilibrium flow' (VE), respectively, hereafter). This has been accomplished to understand how the value of the acoustic surface gravity, which is one of the primary quantifiers of the analogue gravity phenomena, gets influenced by the matter geometry as well as with the equation of state describing the infalling matter.

It is, however, to be noted that most of the astrophysical black holes are believed to be of Kerr type, i.e., they possess non-zero values of the black hole spin parameter (a) Miller et al. 2009], Dauser et al. 2010, Kato et al. 2010], Tchekhovskov et al. 2010|, Ziolkowski 2010|, Buliga et al. 2011], Daly 2011|, Martínez-Sansigre and Rawlings 2011|, McClintock et al. 2011|, Nixon et al. 2011, Revnolds et al. 2012,, Tchekhovskov and McKinnev 2012, Brenneman 2013], Dotti et al. 2013, , McKinnev et al. |2013|, Fabian et al. 2014|, Healv et al. 2014], Jiang et al. 2014], Nemmen and Tchekhovskoy [2014], Sesana et al. 2014]. Hence, it is necessary to understand how the black hole spin dependence of the prominent features of the acoustic geometry gets influenced by the matter geometry. In our present work, which may be considered as the sequel of Tarafdar and Das 2015], we address this issue. In this connection, it is to be noted that purely from astrophysical viewpoint, Tarafdar et al. [2017] have investigated the integral stationary accretion solutions onto a Kerr black hole to understand the multi-transonic structure of such flow and the formation of the stationary shock transitions. In their work, the parameter space spanned by various initial boundary conditions (governing the flow) has been constructed to show how the stationary shocks form for various flow geometries as well as for various equations of state. In summary, the multi-transonic properties of 
general relativistic accretion (adiabatic and isothermal) of matter in the Kerr metric has been thoroughly analyzed in the aforementioned work to understand how the geometric configuration of accretion flow influences the black hole spin dependence of the multi-transonic flow profile for such accretion.

Our present work will take directions from the results obtained by Tarafdar et al. [2017]. We would like to study the variation of the acoustic surface gravity with the black hole spin for multi-transonic shocked accretion solutions in three different matter geometries. We thus identify the regions of the parameter space responsible for shock formation using the formalism introduced in Tarafdar et al. [2017]. Once we identify such parameters, we shall use the respective boundary conditions to calculate the value of the analogue surface gravity in terms of the accretion variables defined at the critical points (see subsequent sections for detailed description of such procedure). We then study the variation of the values of such acoustic surface gravity with the black hole spin. We study such variation for three different geometric configurations of matter to understand how the $\kappa-a$ dependence gets influenced by the matter geometry. We perform such analysis for both polytropic as well as for isothermal accretion.

\section{Outline of the solution scheme}

We consider low angular momentum inviscid accretion onto a Kerr black hole along the equatorial plane. The space-time is described by Boyer-Lindquist line element of the following form (normalized for $G=c=M_{B H}=1$ ), where $G, c$ and $M_{B H}$ are the universal gravitational constant, velocity of light in vacuum and mass of the black hole respectively.

$$
d s^{2}=g_{\mu \nu} d x^{\mu} d x^{\nu}=-\frac{r^{2} \Delta}{A} d t^{2}+\frac{A}{r^{2}}(d \phi-\omega d t)^{2}+\frac{r^{2}}{\Delta} d r^{2}+d z^{2}
$$

where, $\Delta=r^{2}-2 r+a^{2}, A=r^{4}+r^{2} a^{2}+2 r a^{2}, \omega=\frac{2 a r}{A}, a$ being the Kerr parameter.

Following usual procedure, the acoustic surface gravity $\kappa$ may be expressed as,

$$
\kappa=\left[\kappa_{0}\left(\frac{d u}{d r}-\frac{d c_{s}}{d r}\right)\right]_{r=r_{h}}
$$

where $r_{h}$ denotes the location of the acoustic horizon, which actually is the location of the sonic point for a transonic flow, $u$ represents the dynamical radial advective velocity defined on the equatorial plane, $c_{s}$ is the corresponding speed of sound, and

$$
\kappa_{0}=\frac{\left(a^{2}+(r-2) r\right) \sqrt{\left(a^{2}(r+2)+r^{3}-\lambda^{2}(r-2)-4 a \lambda\right)}}{\left(1-c_{s}^{2}\right)\left(a^{2}(r+2)-2 a \lambda+r^{3}\right) \sqrt{r}} .
$$

As is obvious from the above expression, for a certain pre-defined set of initial boundary conditions defined by the specific flow energy $\mathcal{E}$, specific angular momentum $\lambda$, the adiabatic index $\gamma$ and the Kerr parameter $a$, one has to use the 
critical point analysis to find out the critical points and thence, the sonic points by integrating the stationary flow solutions. Once the sonic point is obtained, the value of the advective velocity $u$, the sound speed $c_{s}$ and the respective space gradients of $u$ and $c_{s}$ will be calculated at the sonic points. Such sonic points are actually the acoustic horizons becasue the flow makes a transition from subsonic to supersonic state at those points. Once the set of values of the location of the sonic points $\left(r_{s}\right)$ and $\left[u, c_{s}, \frac{d u}{d r}, \frac{d c_{s}}{d r}\right]_{r_{s}}$ are obtained, $\kappa$ can be calculated for polytropic accretion for a set of initial boundary conditions prescribed by $[\mathcal{E}, \lambda, \gamma, a]$. For isothermal accretion, the initial boundary conditions are specified by $[\lambda, T, a]$, where $T$ is the bulk flow (ion) temperature. Since isothermal sound speed is position independent, the corresponding value of the acoustic surface gravity can be calculated by knowing the location of the isothermal sonic point $r_{s}$ and $\left[u, c_{s}, \frac{d u}{d r}\right]_{r_{s}}$, for a fixed set of values of $[\lambda, T, a]$. Tarafdar et al. [2017] (sections 2-6) describes how to calculate $\left(r_{s},\left[u, c_{s}, \frac{d u}{d r}, \frac{d c_{s}}{d r}\right]_{r_{s}}\right)$ for a particular set of $[\mathcal{E}, \lambda, \gamma, a]$ for polytropic accretion for three different geometric configurations of the flow. Similar calculations are available for isothermal accretion as well (see, e.g. section 9-13 of Tarafdar et al. [2017]).

For certain values of $[\mathcal{E}, \lambda, \gamma, a]$ and $[T, \lambda, a]$, stationary integral solutions corresponding to the adiabatic as well as to isothermal flow becomes multitransonic. That is, the transition from subsonic to supersonic regime takes place twice, leading to the formation of two black hole like acoustic horizons. Flow lines between two such horizons are connected through a stationary shock. At the shock location, an acoustic white hole is formed where the acoustic surface gravity becomes infinite. In principle, what happens is as follows:

At a large distance from the gravitational horizon of the astrophysical black hole, accreting matter remains subsonic. Such subsonic matter encounters an outer sonic point and becomes supersonic. Such supersonic flow may then encounter a stationary shock (depending on whether certain criteria, the general relativistic Rankine-Hugoniot condition for the polytropic flow, for example, are satisfied) and becomes subsonic, The shock transition is a discontinuous transition. $\left[u, c_{s}\right]$ changes discontinuously at the shock location. Hence, $\left[\frac{d u}{d r}, \frac{d c_{s}}{d r}\right]$ diverges at the shock and hence $\kappa$ diverges at the white hole as well, whereas the values of $\kappa$ at the two acoustic black holes, one located at the outer sonic point and the other located at the inner sonic point, remain finite. Section 5 of $\mathrm{Pu}$ et al. 2012] presents the exemplary methodology for the calculation of $\kappa$ for a typical multi-transonic shocked accretion for flow in hydrostatic equilibrium along the vertical direction.

As mentioned in the introduction section that our main goal in this project is to study how the $\kappa-a$ dependence gets influenced by the geometrical configuration of matter. To accomplish this task, one thus needs to have idea about how the shock formation phenomenon is guided by the spin parameter $a$. Also since we need to compare $\kappa-a$ plot for three different matter geometries, it is required to obtain the aforementioned shock parameter space for all three geometries and then concentrate on the mutually overlapping region. 
Following the methodology developed in Tarafdar et al. 2017], we thus construct shock forming parameter space (spanned by $\lambda$ and $a$ keeping $\mathcal{E}$ and $\gamma$ of the flow constant for adiabatic accretion, and by keeping $T$ of the flow constant for isothermal accretion) for the different flow geometries and then identify the common region of overlap. From the identified region, we shall choose the range of $a$ corresponding to a fixed set of $[\mathcal{E}, \lambda, \gamma]$ for adiabatic accretion and $[T, \lambda]$ for isothermal accretion. For those values of $a$, we shall calculate the locations of sonic points $r_{s}$, the shock location $r_{s h}$ and $\left[u, c_{s}, \frac{d u}{d r}, \frac{d c_{s}}{d r}\right]_{r_{s}}$ for the adiabatic flow and $\left[u, c_{s}, \frac{d u}{d r}\right]_{r_{s}}$ for the isothermal flow. The values of $r_{s}, u, c_{s}, \frac{d u}{d r}$ and $\frac{d c_{s}}{d r}$ for various values of $a$ will then be substituted in the respective expression of $\kappa$ as obtained in eqn.(2), and $\kappa-a$ will be obtained for three different geometric configurations of matter.

\section{Dependence of $\kappa$ on $a$ for adiabatic accretion}
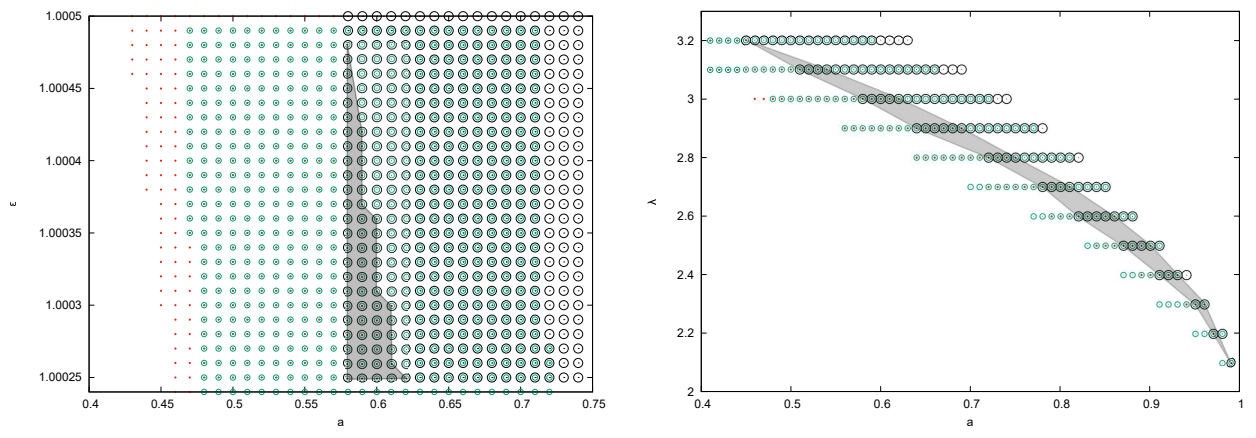

Figure 1: $\mathcal{E}-a(\lambda=3.0)$ and $\lambda-a(\mathcal{E}=1.00025)$ plots for $\gamma=4 / 3$, showing the common shock overlap regions for the three disc geometries. Green circles, red dots and black circles represent $\mathrm{CF}, \mathrm{VE}$ and $\mathrm{CH}$ flows respectively.

In fig. 1 , we plot the parameter space region spanned by $[\mathcal{E}, \lambda]$ and $a$ for which shock forms in all three geometric configurations of accreting matter for a fixed set of values of the remaining parameters $\left(\gamma=\frac{4}{3}\right.$ and $[\lambda=3.0]$ for $\mathcal{E}-a$ diagram, $[\mathcal{E}=1.00025]$ for $\lambda-a$ diagram). One has to understand that we are interested in studying the variation of $\kappa$ with the black hole spin. It is thus necessary that out of all parameters determining the flow, the parameters other than the spin have to be kept constant and $a$ has to be varied to calculate $r_{s}$ and $\left[u, c_{s}, \frac{d u}{d r}, \frac{d c_{s}}{d r}\right]_{r_{s}}$ accordingly for all matter geometries. Hence, which parameter out of the rest is plotted along the y-axis is somewhat immaterial. A common region of shock formation for three different geometries (the shaded regions in fig. 1) can be demonstrated by plotting any of the other parameters against $a$. In the present picture, we have chosen such values of the fixed parameters so as to enhance the range of $a$ for which the common shock forming region forms. Similar figure can be produced by plotting $\gamma$ vs. $a$ as well. 
Note that the multitransonic flow possesses two sonic points, $r_{s}^{i n}$ (inner one, closer to the black hole event horizon) and $r_{s}^{\text {out }}$ (outer one, far away from the event horizon). Thus, we have two different values of the acoustic surface gravity, $\kappa^{\text {in }}$ defined at the inner sonic point, and $\kappa^{\text {out }}$ defined at the outer sonic point. At the shock, the accretion variables change discontinuously and therefore the space derivatives of such variables diverge. Hence, the acoustic surface gravity at the shock location $\kappa^{\text {sh }}$ cannot be computed.
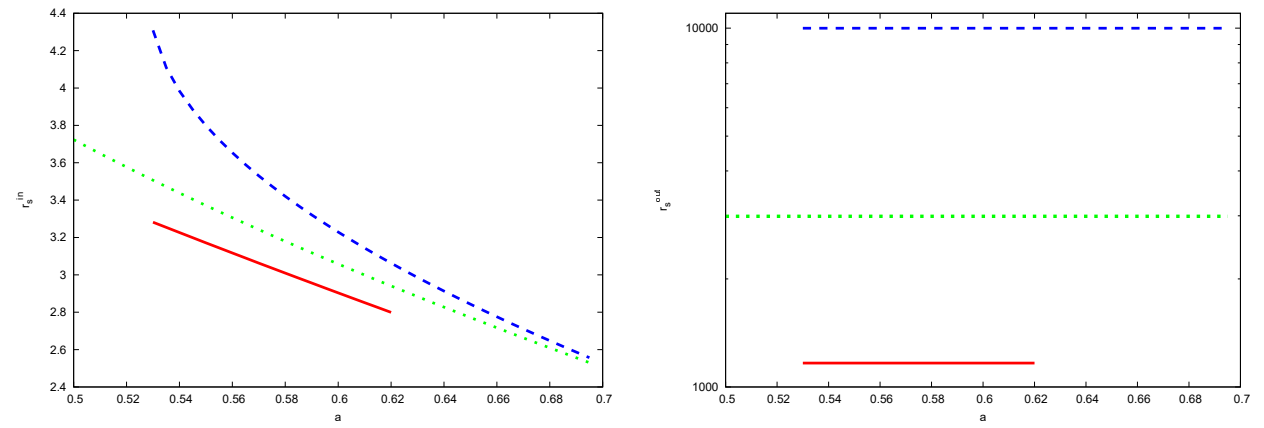

Figure 2: $r_{s}^{\text {in }}-a$ and $r_{s}^{\text {out }}-a(\mathcal{E}=1.00025, \lambda=3.0, \gamma=4 / 3)$ plots. Green dotted line, red solid line and blue dashed line represent $\mathrm{CF}, \mathrm{VE}$ and $\mathrm{CH}$ flows respectively.

In fig. 2, we plot the locations of the inner acoustic horizon $r_{s}^{i n}$ and the outer acoustic horizons $r_{s}^{\text {out }}$, respectively, as functions of $a$, for three different flow geometries. One notes that the influence of the Kerr parameter in changing the values of the acoustic horizon locations, is more prominent for the inner horizons. This is somewhat obvious, since the outer type sonic points form at a reasonably large distance from the black hole where the space time tends to become Newtonian and the black hole spin does not influence the flow properties significantly.

In fig. 3 , we plot the variation of the acoustic surface gravity (evaluated at the inner and at the outer sonic points, respectively) as a function of the black hole spin for three different geometric configurations of matter. As explained in the previous sections, it is first necessary to locate the shock forming region in the parameter space which is common to the three different geometries. Since, only a small portion of the parameter space is obtained through such procedure, hence the $\kappa-a$ relationship is obtained for a limited range of black hole spin, and for all the disc structures, the span of $a$ may not be exactly the same. We find that the numerical value of $\kappa^{i n}$ is always significantly large compared to the corresponding value of $\kappa^{\text {out }}$ for same set of values of $[\mathcal{E}, \lambda, \gamma, a]$. This is, once again, somewhat obvious because the $r_{s}^{\text {in }}$ forms closer to the black hole (compared to $r_{s}^{\text {out }}$ ) and hence at its location gravity becomes stronger. It is also observed that the variation of acoustic surface gravity with black hole spin gets significantly influenced by the geometric configuration of the flow. From fig. 3, 

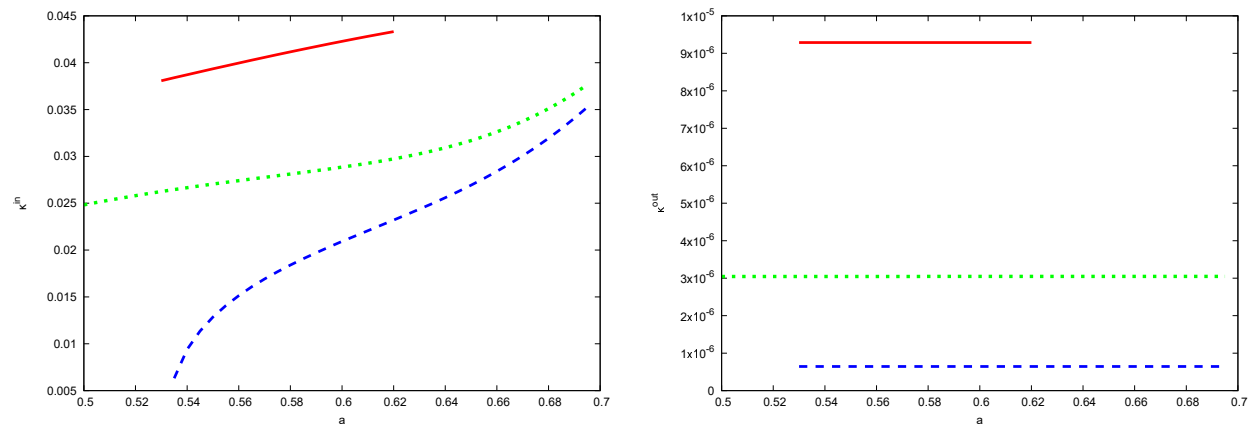

Figure 3: $\kappa^{\text {in }}-a$ and $\kappa^{\text {out }}-a(\mathcal{E}=1.00025, \lambda=3.0, \gamma=4 / 3)$ plots. Green dotted line, red solid line and blue dashed line represent $\mathrm{CF}, \mathrm{VE}$ and $\mathrm{CH}$ flows respectively.

it is observed that

$$
\kappa_{V E}>\kappa_{C F}>\kappa_{C H}
$$

for the same value of black hole spin. However, as of now, by plotting the $\kappa-a$ graph for multitransonic shocked accretion, we have not been able to cover a reasonably large span of the Kerr parameter. We would like to observe how $\kappa$ varies over the entire astrophysically relevant range of $a$ from -1 to +1 .

In order to obtain such a plot, we shift our attention from multitransonic shocked accretion to monotransonic accretion. In case of monotransonic flow, the three different disc structures overlap almost over the entire range of $a$. This task is absolutely essential for us to perform, in order to comment on the global variation of any quantity of our interest (in this case, $\kappa$ ) with black hole spin. It is also interesting to note that such variation covers both prograde and retrograde flows, and thus any asymmetry in the behaviour of the plot on positive and negative sides of the $a$-axis points towards a possible instrument to distinguish between the two different kinds of fluid flow around the rotating central massive compact objects.

In fig. 4, the variation of $\kappa$ with $a$ has been shown for all three flow geometries considering monotransonic accretion. The figure has been obtained for a given set of values for $[\mathcal{E}, \lambda, \gamma]$ corresponding to the stationary monotransonic accretion solutions. It is observed that the constant height disc flow does not have any maxima or point of inflection, whereas the other two flow geometries manifest such peaks. This, however, does not necessarily indicate that the constant height disc flow does not exhibit maxima in general. This artifact is solely attributed to the choice of $[\mathcal{E}, \lambda, \gamma]$ particularly for the given figure. As a matter of fact, all three flow geometries possess extrema in their dependence of $\kappa$ on spin, however, this figure clearly indicates that there is a shift in the position of the peaks with variation of the other flow parameters. 


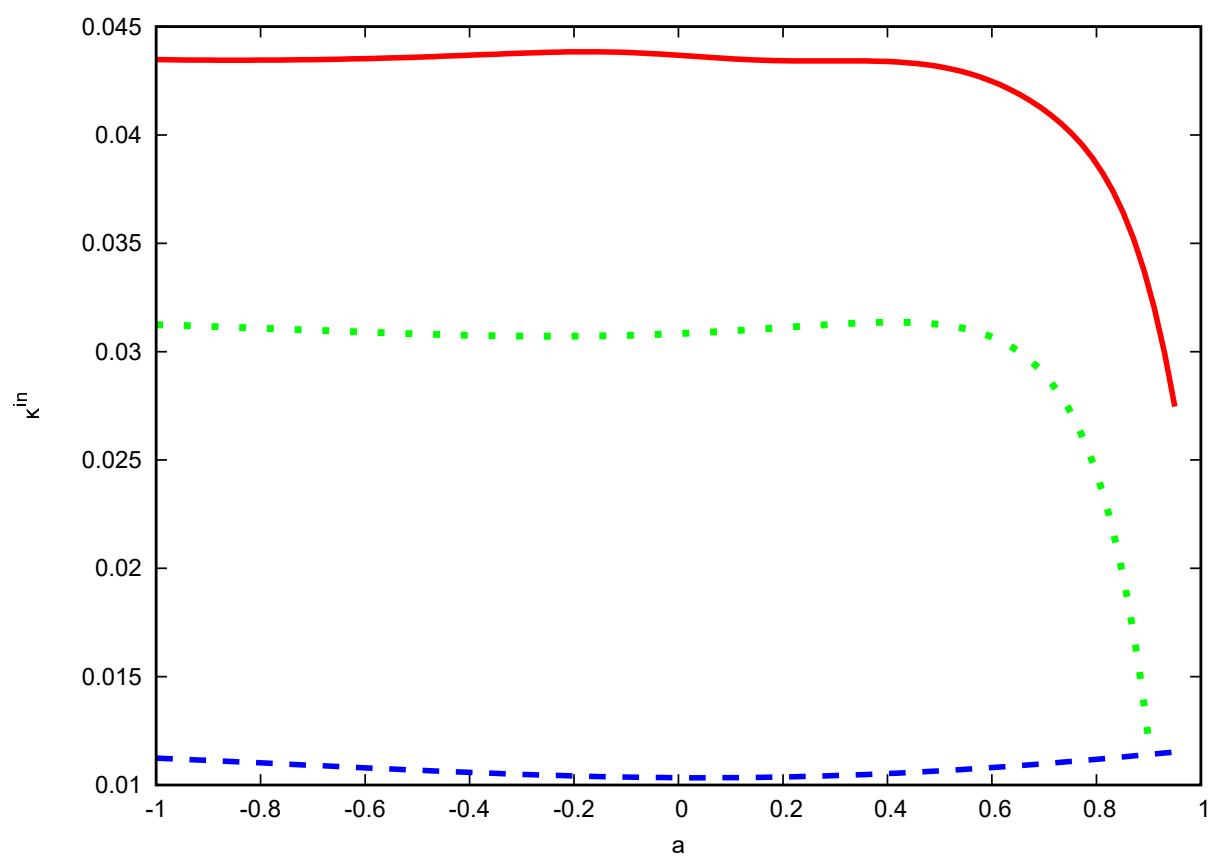

Figure 4: $\kappa^{i n}-a(\mathcal{E}=1.2, \lambda=2.0, \gamma=4 / 3)$ plots for monotransonic accretion. Green dotted line, red solid line and blue dashed line represent $\mathrm{CF}, \mathrm{VE}$ and $\mathrm{CH}$ flows respectively.

\section{Dependence of $\kappa$ on $a$ for isothermal accretion}

In fig. 5, we show the parameter space spanned by the constant flow temperature $(T)$ and the black hole spin to demonstrate the common shock forming region for the different geometric configurations of matter. Similar figures can be produced in the $\lambda-a$ parameter space as well. Following the same line of discussion in the adiabatic section, one may obtain $\kappa^{i n}, \kappa^{\text {out }}, \kappa^{\text {sh }}$ in the isothermal case as well, where $\kappa^{\text {sh }}$ cannot be evaluated since the space gradients of the accretion variables diverge at the shock location.

In fig. 6, spin dependence of the location of the inner and the outer horizons are shown. As expected, $a$ has stronger influence in determining the variation of $r_{s}^{i n}$ in comparision to the variation of $r_{s}^{\text {out }}$.

In fig. 7 , we plot the variation of $\kappa^{\text {in }}$ and $\kappa^{\text {out }}$ with the Kerr parameter. In agreement with the discussions presented in the previous sections, $\kappa^{\text {in }}>>\kappa^{\text {out }}$ for all sets of $[T, \lambda, a]$ also in the case of isothermal accretion. It is also observed that

$$
\kappa_{V E}>\kappa_{C F}>\kappa_{C H}
$$

which is in accord with the observations in the polytropic section. However, the 


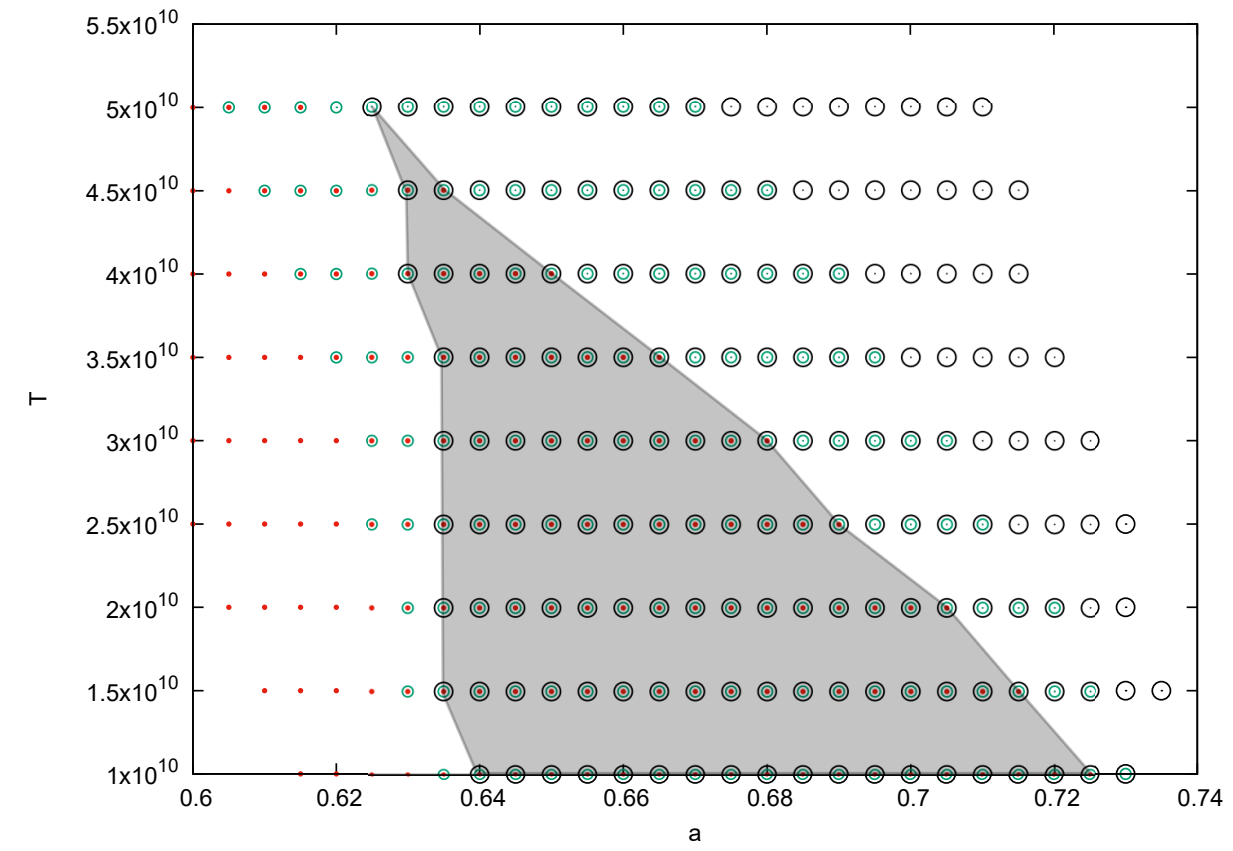

Figure 5: $T-a(\lambda=3.0)$ plot showing the common shock overlap regions for the three disc geometries. $T$ is in units of Kelvin. Green circles, red dots and black circles represent $\mathrm{CF}, \mathrm{VE}$ and $\mathrm{CH}$ flows respectively.
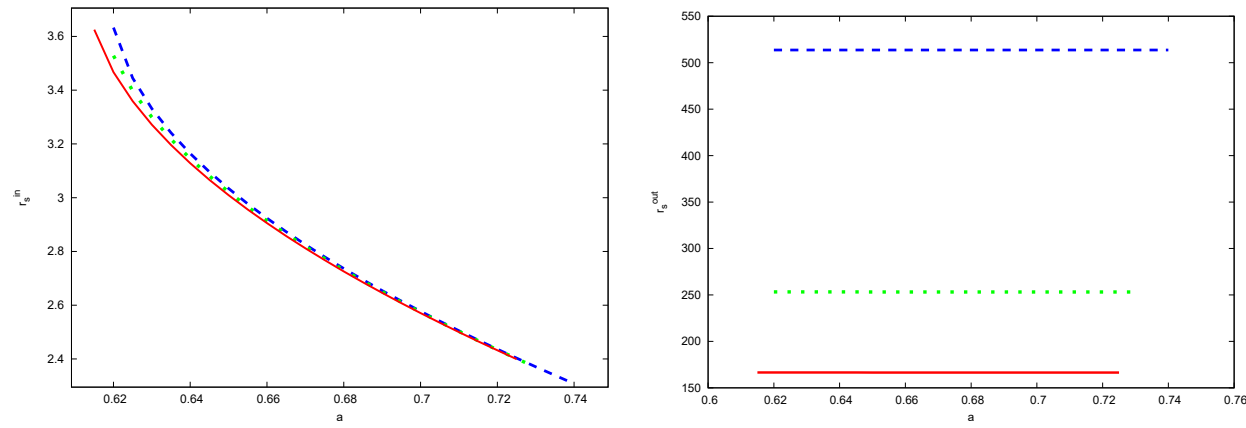

Figure 6: $r_{s}^{\text {in }}-a$ and $r_{s}^{\text {out }}-a\left(T=10^{10} \mathrm{~K}, \lambda=3.0\right)$ plots. Green dotted line, red solid line and blue dashed line represent $\mathrm{CF}, \mathrm{VE}$ and $\mathrm{CH}$ flows respectively.

individual trend of variation of $\kappa^{i n}$ with $a$ for the vertical hydrostatic equilibrium disc is found to be contrary to those of the other two disc models. In this context, we must remember that multitransonic shocked accretion solutions offer a very narrow window of spin in which all the disc geometries overlap. Hence, behaviour of a physical quantity in the given small range of $a$ may not be quite a reliable representation of the bigger picture. Thus, in order to resolve this 

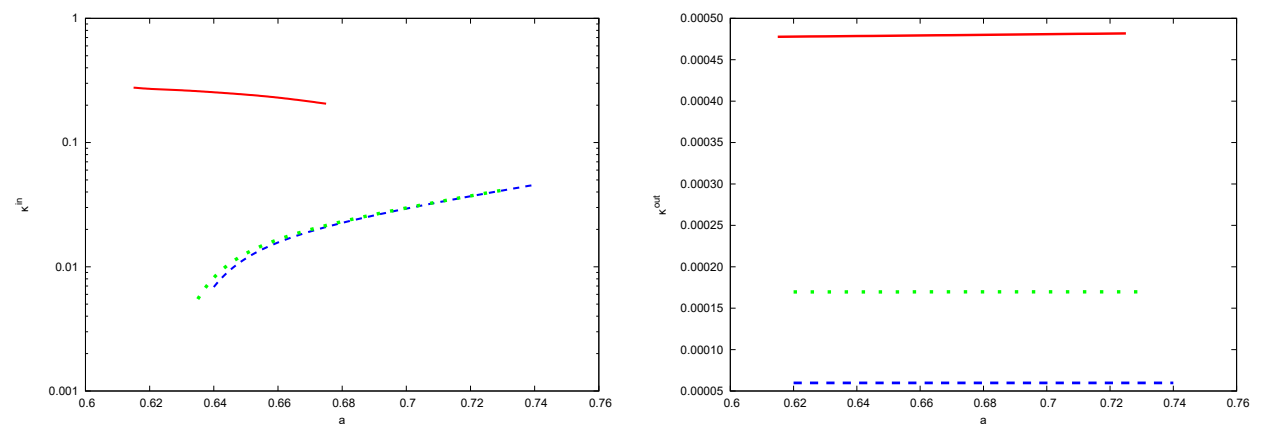

Figure 7: $\kappa^{\text {in }}-a$ and $\kappa^{\text {out }}-a\left(T=10^{10} \mathrm{~K}, \lambda=3.0\right)$ plots. Green dotted line, red solid line and blue dashed line represent $\mathrm{CF}, \mathrm{VE}$ and $\mathrm{CH}$ flows respectively.

discrepancy, we need to look at the global variation of acoustic surface gravity over the entire span of Kerr parameter.

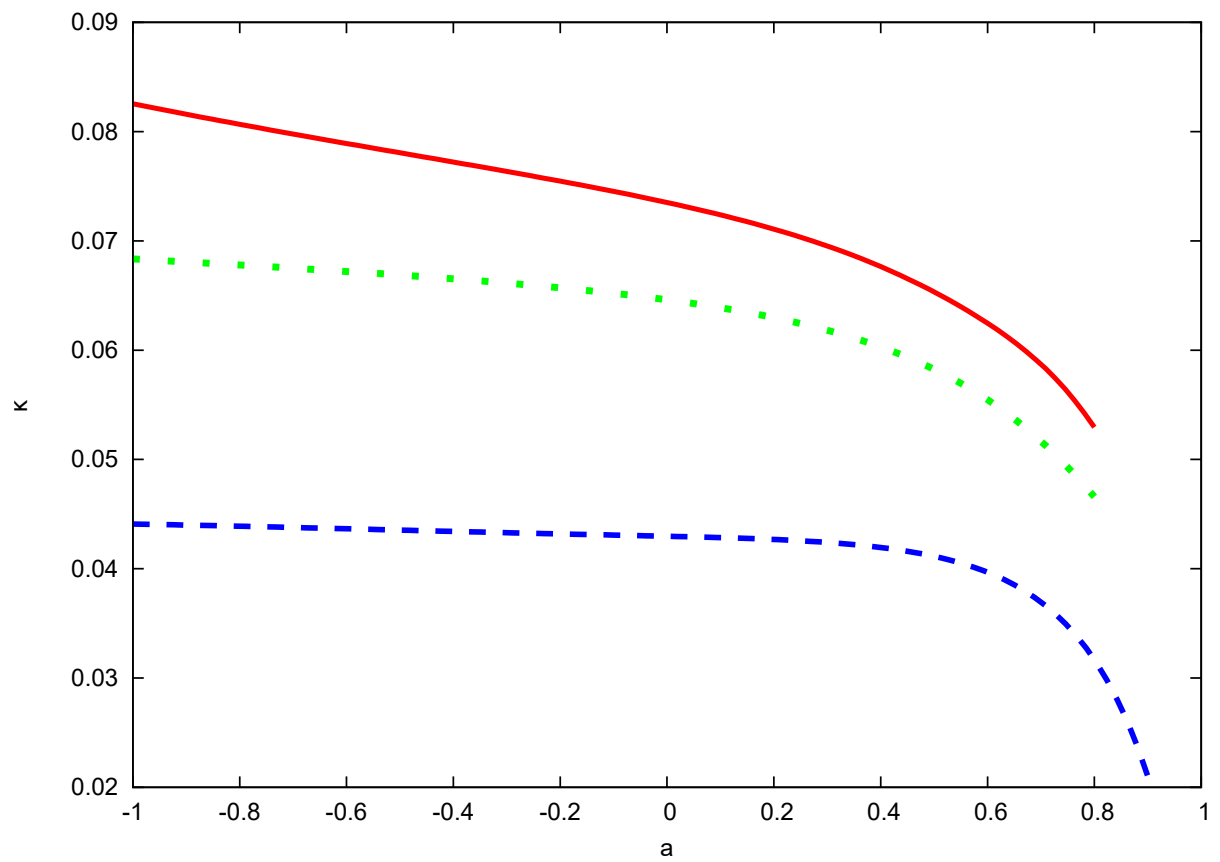

Figure 8: $\kappa-a\left(T=10^{12} \mathrm{~K}, \lambda=2.0\right)$ plot for monotransonic isothermal accretion. $T$ is in units of Kelvin. Green dotted line, red solid line and blue dashed line represent $\mathrm{CF}, \mathrm{VE}$ and $\mathrm{CH}$ flows respectively.

As discussed in the previous section, the only way to have a glimpse of the global dependence of a variable on flow parameters is to study the corresponding dependence for monotransonic accretion solutions. Fig. 8 depicts the global 
variational trend of $\kappa$ over the entire range of black hole spin for monotransonic isothermal accretion. It is observed that the trend of the dependence of $\kappa$ on the disc geometry remains the same as for polytropic accretion. From the figure, it is clear that a seemingly anti-correlating behaviour amongst the various flow configurations, may actually be a manifestation of constrained observations over limited ranges of parameters allowing for a desired class of solutions.

\section{Concluding remarks}

At this stage, it is imperative to reiterate the basic motivation behind the work presented in this manuscript. Hawking radiation is universal in the sense that the radiation does not depend on the medium. On the contrary, analogue effects depend on the physical properties of matter and such dependence gets reflected through the corresponding dispersion relation, see, e.g., Leonhardt and Robertson 2012], Robertson 2012] for further details. In existing works, however, the background space-time has been considered to be flat Minkowskian, and the configuration of the fluid (whether classical or quantum) has been assumed to follow certain simplified symmetry, like planar, circular, or spherical, for instance Barceló et al. [2005]. Our work is way more involved in this context. Firstly, the space-time in which the dynamics of the fluid has been defined, is itself curved since we study the fluid motion in general relativistic black hole metric. Also such curved space-time has its variant - it may be of Schwarzschild or of Kerr type - where the specific unique characteristic feature of such different background geometries will determine the nature of the embedded acoustic geometry. In addition to the geometry of the space-time, the geometry of the fluid itself determines the features of the sonic geometry. In the present work, we have shown how the behaviour of the analogue acoustic geometry is determined by the combined effect of the space-time geometry as well as the matter geometry. Such an attempt provides rich features of the sonic geometry which has been demonstrated in this work through the dependence of the acoustic surface gravity on both the space-time and the matter geometry. We have also shown which disc structure may produce maximum value of the analogue temperature (which is a scalar multiple of $\kappa$ ).

\section{Acknowledgments}

PT would like to acknowledge the kind hospitality provided by HRI, Allahabad, India, for several visits through the XII ${ }^{\text {th }}$ plan budget of Cosmology and High Energy Astrophysics grant. PT intends to acknowledge Md. Arif Shaikh for pointing out crucial corrections in the manuscript. The authors also intend to acknowledge the anonymous reviewer for useful comments and suggestions. 


\section{References}

H. Abraham, N. Bilić, and T. K. Das. Classical \&3 Quantum Gravity, 23:2371, 2006.

Carlos Barceló, Stefano Liberati, and Matt Visser. Analogue gravity. Living Reviews in Relativity, 8(1):12, 2005. ISSN 1433-8351. doi: 10.12942/lrr-2005-12. URL http://dx.doi.org/10.12942/lrr-2005-12

N. Bilić, A. Choudhary, T. K. Das, and S. Nag. Classical \& Quantum Gravity, 31:35002, 2014.

L. Brenneman. Measuring the Angular Momentum of Supermassive Black Holes, Springer Briefs in Astronomy. Springer-Verlag, New York, 2013.

S. D. Buliga, V. I. Globina, Y. N. Gnedin, T. M. Natsvlishvili, M. Y. Pitrovich, and N. A. Shakht. Astrophysics, 54(4):548, 2011.

R. A. Daly. MNRAS, 414:1253, 2011.

T. K. Das, N. Bilic, and S. Dasgupta. A black-hole accretion disc as an analogue gravity model. JCAP, 6:009, June 2007. doi: 10.1088/1475-7516/2007/06/ 009.

T. Dauser, J. Wilms, C. S. Reynolds, and L. W. Brenneman. MNRAS, 409: $1534,2010$.

M. Dotti, M. Colpi, S. Pallini, A. Perego, and M. Volonteri. ApJ, 762(2):10, 2013.

A. C. Fabian, M. L. Parker, D. R. Wilkins, J. M. Miller, E. Kara, C. S. Reynolds, and T. Dauser. MNRAS, 439:2307, 2014.

J. Healy, C. Lousto, and Y. Zlochower. arXiv:1406.7295 [gr-qc], 2014.

J. Jiang, C. Bambi, and J.F. Steiner. arXiv:1406.5677 [gr-qc], 2014.

Y. Kato, M. Miyoshi, R. Takahashi, H. Negoro, and R. Matsumoto. MNRAS, 403:L74, 2010.

Ulf Leonhardt and Scott Robertson. Analytical theory of hawking radiation in dispersive media. New Journal of Physics, 14, 5 2012. ISSN 1367-2630. doi: 10.1088/1367-2630/14/5/053003.

A. Martínez-Sansigre and S. Rawlings. MNRAS, 414:1937, 2011.

J. E. McClintock, R. Narayan, S. W. Davis, L. Gou, A. Kulkarni, J. A. Orosz, R. F. Penna, R. A. Remillard, and J. F. Steiner. Classical and Quantum Gravity, 28(11):114009, 2011.

J. C. McKinney, A. Tchekhovskoy, and R. D. Blandford. Science, 339:49, 2013. 
J. M. Miller, C. S. Reynolds, A. C. Fabian, G. Miniutti, and L. C. Gallo. ApJ, 697:900-912, 2009.

R. Nemmen and A. Tchekhovskoy. arXiv:1406.7420 [astro-ph.HE], 2014.

C. J. Nixon, P. J. Cossins, A. R. King, and J. E. Pringle. MNRAS, 412:1591, 2011.

H. Y. Pu, I. Maity, T. K. Das, and H. K. Chang. Classical \& Quantum Gravity, 29:245020, 2012.

C. S. Reynolds, L. W. Brenneman, A. M. Lohfink, M. L. Trippe, J. M. Miller, R. C. Reis, M. A. Nowak, and A. C. Fabian. AIP Conference Proceedings, 1427:157-164, 2012.

S. J. Robertson. The theory of Hawking radiation in laboratory analogues. Journal of Physics B Atomic Molecular Physics, 45(16):163001, August 2012. doi: 10.1088/0953-4075/45/16/163001.

A. Sesana, E. Barausse, M. Dotti, and E. M. Rossi. arXiv:1402.7088 [astroph.CO], 2014.

P. Tarafdar and T. K. Das. IJMPD, 24:1550096, 2015.

P. Tarafdar, D. A. Bollimpalli, S. Nag, and T. K. Das. arXiv:1612.06882, 2017.

A. Tchekhovskoy and J. C. McKinney. MNRAS, 423(1):L55, 2012.

A. Tchekhovskoy, R. Narayan, and J. C. McKinney. ApJ, 711:50, 2010.

W. Unruh and R. Schützhold. (ed) Quantum Analogues: From Phase Transitions to Black Holes and Cosmology, Lecture Note in Physics, Vol. 718, pg. 1-298. Springer, Berlin, Heidelberg, 2007.

W. G. Unruh. Experimental black-hole evaporation. Physical Review Letters, 46:1351-1353, May 1981. doi: 10.1103/PhysRevLett.46.1351.

M. Visser. Acoustic black holes: horizons, ergospheres and Hawking radiation. Classical and Quantum Gravity, 15:1767-1791, June 1998. doi: 10.1088/ 0264-9381/15/6/024.

J. Ziolkowski. Memorie della Societ'A Astronomica Italiana, 81:294, 2010. 